\title{
Sapho Syndrome in Childhood
}

Helena Munivrana Skvorc ${ }^{1 *}$, Marko Skvorc ${ }^{2}$, Iva Mrkic Kobal ${ }^{3}$, Marija Šenjug Perica ${ }^{4}$, Lana Tambić Bukovac ${ }^{5}$

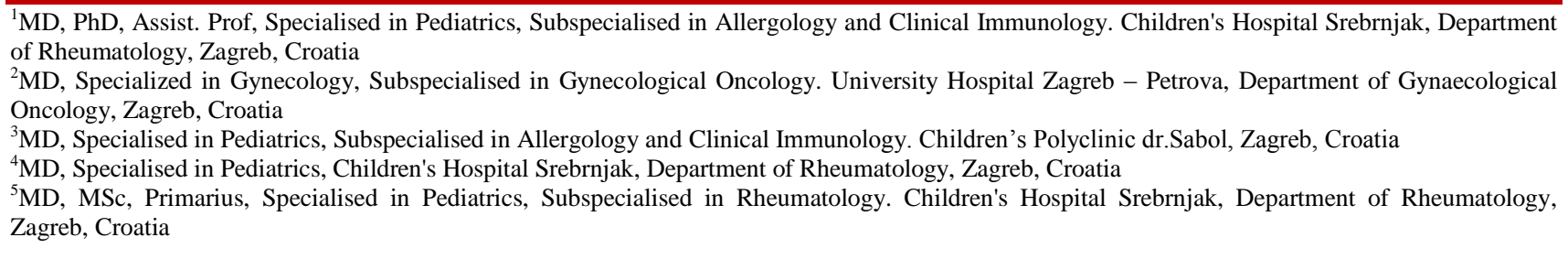

DOI: $10.36347 /$ sjmcr.2021.v09i01.009

| Received: 27.12.2020 | Accepted: 11.01.2021 | Published: 14.01.2021

*Corresponding author: Helena Munivrana Skvorc

Abstract

SAPHO syndrome is an acronym for "Synovitis, Acne, Pustulosis, Hyperostosis and Osteitis". It is combination of cutaneous and musculoskeletal manifestations, such as osteoarthritis and hyperostosis of bones of the anterior chest wall associated with acne fulminans and hidradenitis suppurativa. We present the case of a 15-year old boy with osteoarthritis of the left hip, osteitis of the left carpal bones and right clavicula with acne conglobata. Bone biopsy of the left hip has been carried out and Propionibacterium acnes was found in the bone lesion. Identification of the Propionibacterium acnes from bone biopsy in SAPHO syndrome has occasionally been reported, which could suggest that microorganisms could be a trigger for osteitis and hyperostosis and may play the role in the pathogenesis of the disease. Our patient was treated with nonsteroidal anti-inflammatory drugs, antibiotics and a corticosteroids, which resulted in clinical improvement, but not complete remission, so we introduced methotrexate in therapy. The purpose of this case study is to raise awareness to a set of clinical features of SAPHO syndrome and its early recognition and prompt therapy.

Keywords: SAPHO syndrome, osteoarthritis, Propionibacterium acnes.

Copyright (C) 2021 The Author(s): This is an open-access article distributed under the terms of the Creative Commons Attribution 4.0 International License (CC BY-NC 4.0) which permits unrestricted use, distribution, and reproduction in any medium for non-commercial use provided the original author and source are credited.

\section{INTRODUCTION}

The SAPHO syndrome is an acronym for "synovitis, acne, pustulosis, hyperostosis and osteitis". It is a combination of cutaneous and musculoskeletal manifestations, such as osteoarthritis and hyperostosis of the bones of the anterior chest wall in combination with acne fulminans and hidradenitis suppurativa.

The classic cutaneous manifestations are usually severe acne, which can occur as acne conglobate, acne fulminans or hidradenitis suppurativa. These lesions can occur before, at the same time or after osteoarticular manifestations. Pustular psoriasis has also been described as a manifestation of SAPHO, which may indicate a possible link between psoriatic arthritis (PsA) and SAPHO [1].

Osteoarticular manifestations usually involve the clavicles, sternum, sternocostoclavicular joints, mandible and pelvis. Peripheral arthritis usually involves the knees, hips and ankles [2]. The typical presentation in the paediatric population is CRMO
(Chronic Recurrent Multifocal Osteomyelitis) [2]. In CRMO, the location of the inflammation is more often seen in the extremities than in the axial skeleton, jaw or sternum [3].

There are studies suggesting that microorganisms may be a trigger for osteitis and hyperostosis and may play a role in the pathogenesis of the disease [4]. Occasionally, the identification of Propionibacterium acnes from bone biopsies in SAPHO syndrome has been reported, which may indicate its possible etiological role [5] and a possible role of antibiotic therapy in the treatment of SAPHO [6].

SAPHO is a rare disease with a prevalence of 1 in 10,000 , affecting mainly adults aged 30 to 50 years, although it has been observed at the age of 15 months [7]. In some studies the gender distribution was approximately equal, while in other studies the female prevalence seemed to be in the majority [8, 9]. Geographically, European reports are the most common [10]. 
Helena Munivrana Skvorc et al., Sch J Med Case Rep, Jan, 2021; 9(1): 34-38

This is a rare syndrome and the purpose of this case study is to raise awareness of a number of clinical features of SAPHO syndrome and its early detection and immediate treatment.

\section{CASe Report}

The patient is a 17-year-old boy who came to the orthopedic clinic two years ago for an examination of his left sacroiliac joint pain (SI). The pain in his left hip was present for two months before the examination, and he occasionally complained of pain in the left palm of his hand. He had acne conglobata in his face, back and chest (Figure 1). Skin manifestations occurred 2 years before the skeletal manifestations occurred. He had no significant personal or family history.

The inflammatory parameters were high. Magnetic resonance imaging (MRI) showed subcortical bone marrow edema in the left SI joint, strongly suggesting sacroiliitis and in one trochanter major indicating osteitis. The technetium99 bone image showed a large trochanter on the left side and an active image on the radial side of the left carpal bone. An open biopsy of the left greater trochanter was performed; the pathological examination showed chronic, non-specific inflammatory changes, and in cultures Propionibacterium acnes was isolated. After the biopsy he was discharged home under antibiotic therapy (clindamycin and cefuroxime axetil) and acetylsalicylic acid. A month later he returned for re-evaluation and, as there was no improvement, he was sent to Rheumatology Department where he was hospitalized with the diagnosis of chronic osteomyelitis. During his stay in hospital we started treatment with Clindamicin,
Gentamicin and NSAID. Despite the therapy he developed pain, tenderness and swelling of the right clavicle. The conventional x-ray showed a hyperostosis at the right clavicle. The bone scan Control Technetium99 showed an active image on the medial half of the right clavicle, while the active image on the left greater trochanter was less active. A discrete active image was also found in the right calcaneus and dorsum of the right foot (Figure-2). Since our patient had osteoarticular manifestations with acne conglobata, the diagnosis of SAPHO syndrome was made according to the diagnostic criteria proposed by Kahn. We started treatment with corticosteroids, ibuprofen and pantoprazole. During the 5 days we also prescribed azithromycin for its antimicrobial, anti-inflammatory and immunomodulatory effects. The symptoms and laboratory evaluation improved after starting this therapy and the patient went home. After one year of treatment with corticosteroids, NSAID and azithromycin every few months, there were no expectant remissions of symptoms, no inflammatory parameters of the serum and no imaging findings (Figure-3). Therefore, we started treatment with a disease-modifying antirheumatic (methotrexate), which is still effective today. We succeeded in reducing the dose of corticosteroid; its inflammatory findings in the laboratory improved, pain and swelling of the right clavicle are less pronounced and skin lesions have improved (Figure-4).

During his treatment we consulted a dermatologist and his treatment included topical tetracyclines and retinoins.

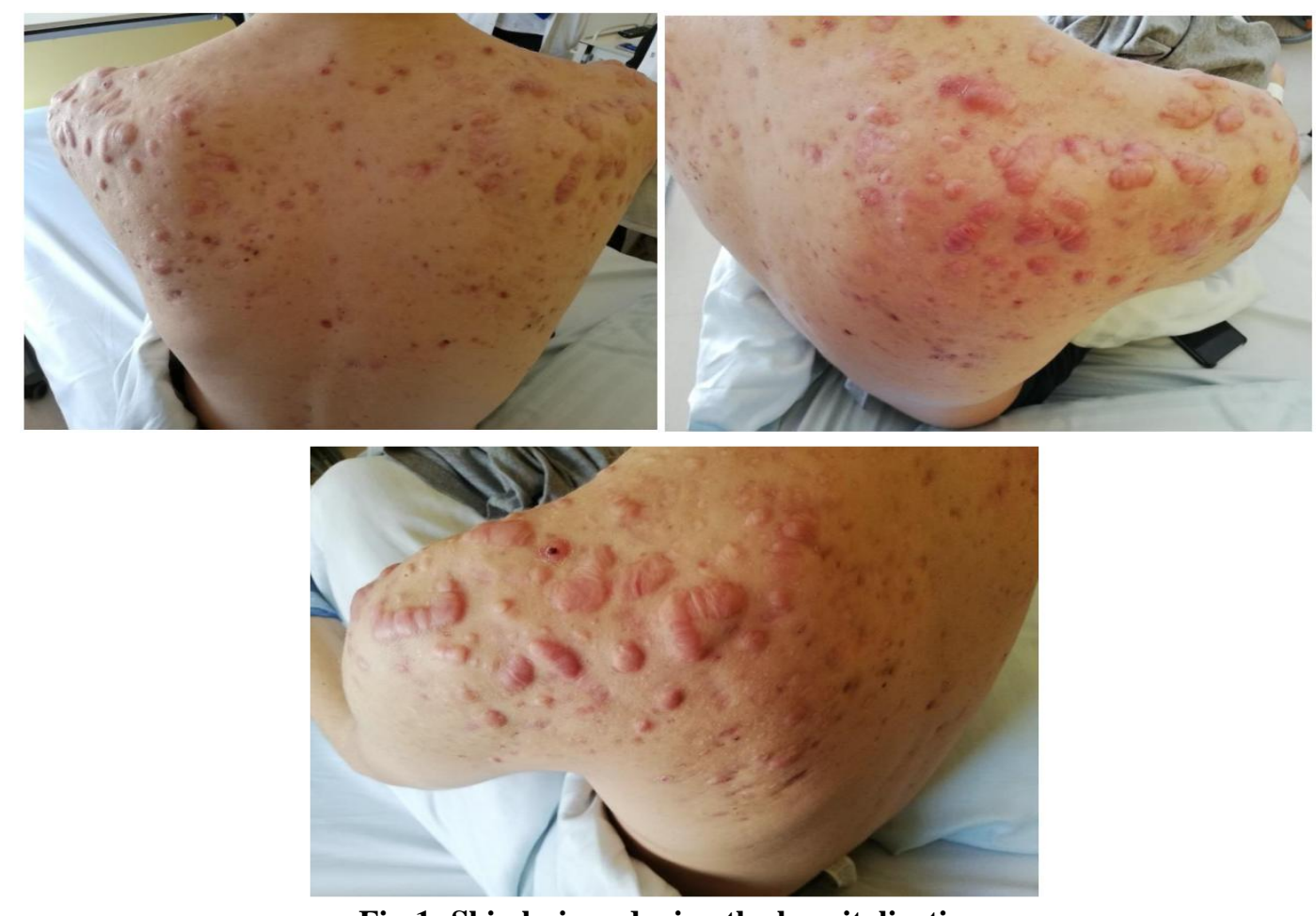

Fig-1: Skin lesions during the hospitalization 
Helena Munivrana Skvorc et al., Sch J Med Case Rep, Jan, 2021; 9(1): 34-38

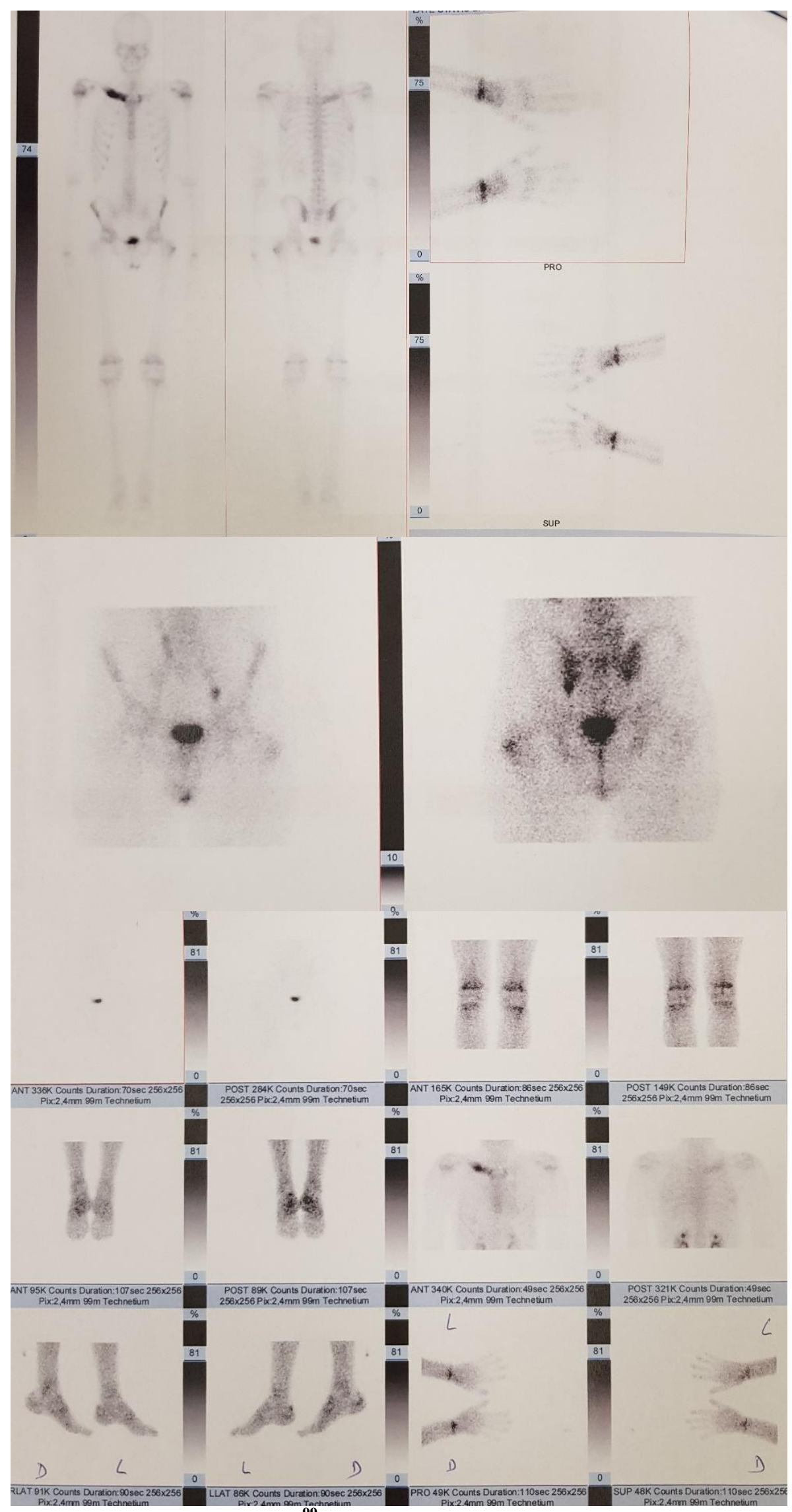

Fig-2: Technetium ${ }^{99}$ bone scan during the hospitalization 


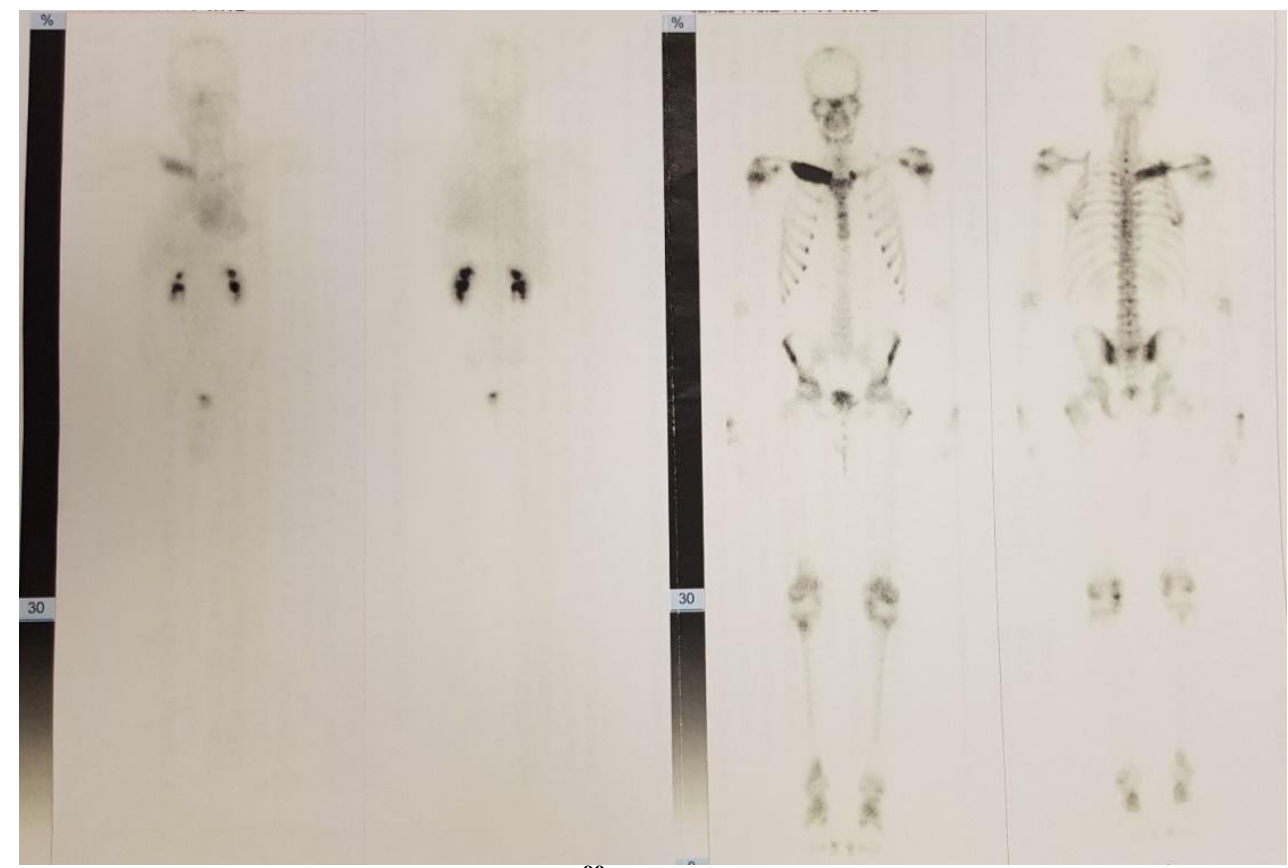

Fig-3: Control Technetium ${ }^{99}$ bone scan after the hospitalization
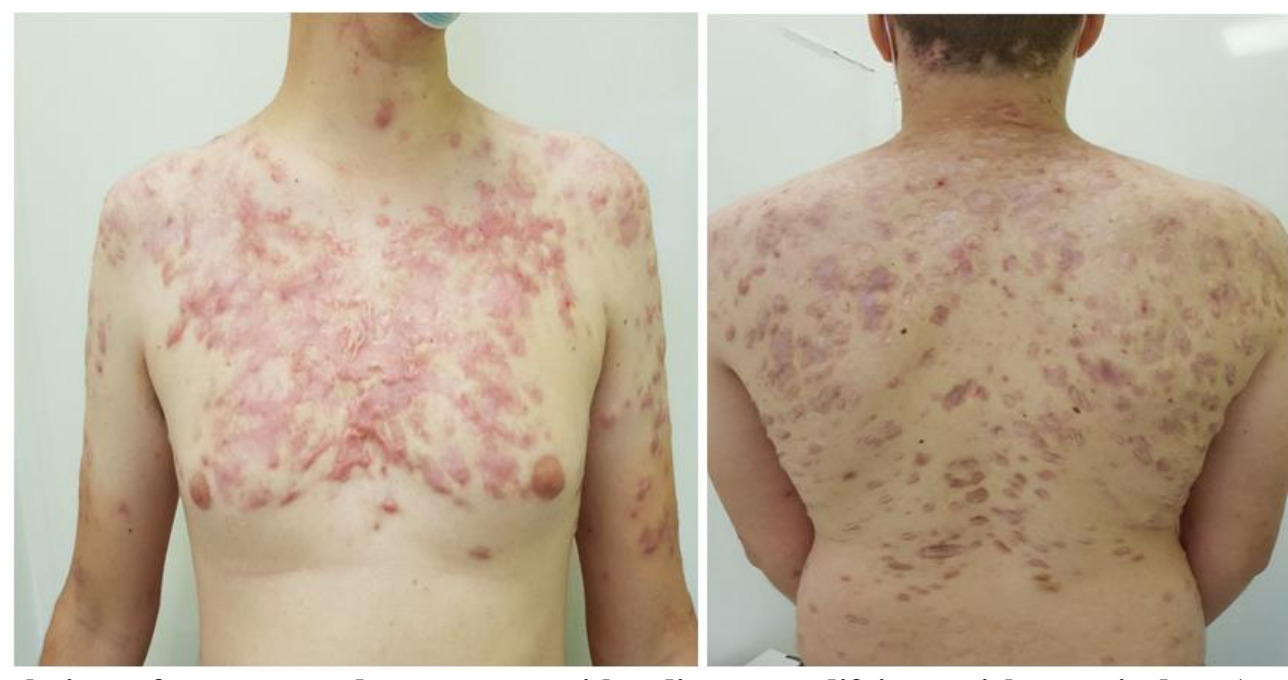

Fig-4: Skin lesions after we started a treatment with a disease-modifying antirheumatic drug (methotrexate)

\section{DISCUSSION}

The SAPHO syndrome is a rare, chronic and recurring disease. It is an autoinflammatory disease associated with increased release of several inflammatory cytokines and global neutrophil activation. The etiopathogenic mechanism includes immunological, genetic and bacteriological factors [11]. Identification of Propionibacterium acnes from bone biopsies in SAPHO syndrome has been reported occasionally, which may indicate its possible etiological role [5]. Propionibacterium acnes is believed to induce auto-amplification of the inflammatory response in genetically determined individuals [11]. In our patient, an open biopsy of the left greater trochanter was obtained and Propionibacterium acnes was isolated, which could be a trigger for the development of the disease.
The diagnosis of SAPHO syndrome is based on the combination of cutaneous and musculoskeletal manifestations and characteristic imaging results (x-ray, MRI, bone scintigraphy). The criteria suggested by Kahn are the most frequently used. Inclusion criteria are osteoarticular involvement associated with palmoplantar pustulosis, osteoarticular involvement associated with severe acne, sterile hyperostosis/osteitis (adults) except for the growth of propionibacterium acnes, chronic recurrent multifocal osteomyelitis (children), and osteoarticular involvement associated with chronic bowel disease. Exclusion criteria are infectious osteitis, tumor-like conditions of the bone and non-inflammatory condensing lesions of the bone [12]. 
Helena Munivrana Skvorc et al., Sch J Med Case Rep, Jan, 2021; 9(1): 34-38

Current treatments for this disease include NSAIDs, anti-inflammatory drugs such as colchicine, corticosteroids and biphosphonates, and diseasemodifying agents such as methotrexate, sulfasalazine and infliximab [13]. There is also a possible role of antibiotic therapy in the treatment of SAPHO [6]. It usually includes azithromycin, doxycycline, sulfamethoxazole/trimetoprim and clindamycin [14]. After the diagnosis was made, our patient was treated with NSAID, corticosteroids and antibiotic therapy. Due to the lack of disease regression, we included methotrexate in the therapy. For the time being the results are encouraging.

The course of the disease is variable. The minority of patients has a self-limited course and most patients have a relapsing-remitting course or a chronic pattern. The factors identified as predictors of a chronic pattern are the female gender, involvement of the anterior chest wall, peripheral arthritis, skin lesions and high concentrations of acute phase reactants at the time of diagnosis [15]. The prognosis is relatively good, although rheumatic manifestations are hardly progressive in most patients [16].

\section{CONCLUSION}

The purpose of this case study is to raise awareness of a number of clinical features of SAPHO syndrome and its early detection and immediate treatment to prevent disease progression. Further studies are needed to better understand the disease and its treatment.

\section{REFERENCE}

1. Kahn MF, Khan MA. The SAPHO syndrome. Baillieres Clin Rheumatol. 1994; 8:333- 62.

2. Earwaker JW, Cotten A. SAPHO: syndrome or concept? Imaging findings. Skeletal Radiol. 2003; 32: $311-27$.

3. Beretta-Piccoli BC, Sauvain MJ, Gal I, Schibler A, Saurenmann T, Kressebuch H, Bianchetti MG. Synovitis, acne, pustulosis, hyperostosis, osteitis (SAPHO) syndrome in childhood: a report of ten cases and review of the literature. European journal of pediatrics. $2000 \mathrm{Jul}$ 1;159(8):594-601.

4. Kahn MF. Why the "SAPHO" syndrome? J Rheumatol. 1995; 22:2017-9.
5. Edlund E, Johnsson U, Lidgren LA, Pettersson H, Sturfelt G, Svensson B, Theander J, Willen H. Palmoplantar pustulosis and sternocostoclavicular arthro-osteitis. Annals of the rheumatic diseases. 1988 Oct 1;47(10):809-15.

6. Colina M, Trotta F. Antibiotics may be useful in the treatment of SAPHO syndrome. Mod Rheumatol. 2014;24(4):697. Epub 2014 Feb 7.

7. Rukavina I. SAPHO syndrome: a review. J Child Orthop. 2015; 9(1):19-27.

8. Nguyen MT, Borchers A, Selmi C, Naguwa SM, Cheema G, Gershwin ME. The SAPHO syndrome. Semin Arthritis Rheum. 2012;42(3):254-265.

9. Mari A, Morla A, Melero M, Schiavone R, Rodriguez J. Diffuse sclerosing osteomyelitis (DSO) of the mandible in SAPHO syndrome: a novel approach with anti-TNF therapy. Systematic review. J Craniomaxillofac Surg. 2014;42(8):1990-1996.

10. Carneiro S, Sampaio-Barros PD. SAPHO syndrome. Rheumatic Disease Clinics. 2013 May 1;39(2):401-18.

11. Hayem G. Valuable lessons from SAPHO syndrome. Joint Bone Spine. 2007; 74:123-126.

12. Hayem G. SAPHO syndrome. Rev Prat. 2004; 54:1635-1636.

13. Olivieri I, Padula A, Ciancio G, Salvarani C, Niccoli L, Cantini F. Successful treatment of SAPHO syndrome with infliximab: Report of two cases Ann Rheum Dis. 2002; 61:375-6.

14. Assmann G, Kueck O, Kirchhoff T, Rosenthal H, Voswinkel J, Pfreundschuh M, Zeidler H, Wagner AD. Efficacy of antibiotic therapy for SAPHO syndrome is lost after its discontinuation: an interventional study. Arthritis research \& therapy. 2009 Oct 1;11(5):R140.

15. Colina M, Govoni M, Orzincolo C, Trotta F. Clinical and radiologic evolution of synovitis, acne, pustulosis, hyperostosis, and osteitis syndrome: a single center study of a cohort of 71 subjects. Arthritis Care \& Research: Official Journal of the American College of Rheumatology. 2009 Jun 15;61(6):813-21.

16. Hayem G, Bouchaud-Chabot A, Benali K, Roux S, Palazzo E, Silbermann-Hoffman O, Kahn MF, Meyer O. SAPHO syndrome: a long-term followup study of 120 cases. InSeminars in arthritis and rheumatism 1999 Dec 1 (Vol. 29, No. 3, pp. 159171). WB Saunders. 